\title{
Comportamiento métrico de las patentes concedidas en Cuba: su contribución a la innovación tecnológica nacional
}

\author{
Maidelyn Díaz Pérez \\ Raudel Giráldez Reyes* \\ Humberto Andrés Carrillo-Calvet ${ }^{\star \star}$
}

Artículo recibido:

28 de julio de 2016

Artículo aceptado:

16 de enero de 2017

\section{RESUMEN}

Los estudios métricos de patentes desde finales del pasado XX son una valiosa herramienta de vigilancia científica tecnológica y de innovación, convirtiéndose en instrumento indispensable para conocer el comportamiento tecnológico internacional. Sin embargo, los estudios patentométricos no son aplicados óptimamente por todos los países, ni por todos los organismos internacionales, tampoco son aprovechadas todas las potencialidades que ofrecen estos estudios para conocer los diferentes contextos de las innovaciones tecnológicas de un país. Este artículo tiene como objetivo analizar

* Universidad de Pinar del Río, Cuba. maidelyn@upr.edu.cu, giraldez@upr.edu.cu.

** Facultad de Ciencias, UNAM . México. carr@servidor.unam.mx.

INVESTIGACIÓN BIBLIOTECOLÓGICA, Número Especial de Bibliometría, 2017, México, ISSN: 2448-8321. pp 271-289 
el comportamiento métrico de las patentes concedidas en Cuba, aplicando una metodología propia que describe las principales innovaciones científico-tecnológicas patentadas por la Oficina Cubana de Propiedad Industrial. La metodología propuesta utiliza el software proIntec para la descarga, normalización, procesamiento, análisis y visualización de los datos procedentes de las patentes, y se aplica un amplio grupo de indicadores métricos relacionales y complejos, así como técnicas de redes sociales para visualizar los principales comportamientos de las innovaciones tecnológicas cubanas. Los resultados finales manifiestan las potencialidades de los estudios métricos de patentes, al poder representar los desarrollos tecnológicos del país y sus contribuciones al sistema de ciencia e innovación tecnológica nacional.

Palabras clave: Estudio patentométrico de innovaciones tecnológicas; Redes de dominios tecnológicos; Indicadores de colaboración tecnológica.

Metric behavior of patents granted in Cuba: its contribution to the national technological innovation Maidelyn Díaz Pérez, Raudel Giráldez Reyes and Humberto Andrés Carrillo-Calvet

\section{Abstract}

Metric patent studies since the end of the last century are a valuable tool for scientific technological and innovation surveillance, becoming an indispensable instrument for knowing the international technological behavior. However, patentometric studies are not applied optimally by all countries or by all international organizations, nor are all the potential of these studies used to know the different contexts of a country's technological innovations. This research aims to analyze the metric behavior of patents granted in Cuba applying an own methodology that describes the main technological scientific innovations patented by the Cuban Office of Industrial Property. The proposed methodology uses 
proIntec software for the download, normalization, processing, analysis and visualization of data from patents, and applies a large group of relational and complex metrics, as well as social networking techniques to visualize the main behaviors of Cuban technological innovations. The final results show the potential of metric patent studies to represent the country's technological developments and its contributions to the national science and technological innovation system.

Keywords: Patentometric studies of technological innovations; Technological domain networks; Technological collaboration indicators.

\section{INTRODUCCIÓN}

— sta investigación se enmarca en una de las modalidades más polémicas de Cla propiedad industrial, la información de patentes, y defiende la premisa que plantea que los estudios de Análisis de Dominios Tecnológicos basados en documentos de patentes son una propuesta factible de los países menos desarrollados para tener una mayor autonomía sobre los conocimientos que se patentan en sus estados (Díaz, 2005; 2007; Díaz y Moya, 2008; Díaz, Rivero y Moya, 2010). El análisis métrico de patentes es una herramienta imprescindible para monitorear, controlar, evaluar y mantener una vigilancia científico tecnológica competitiva de los resultados generados en actividades de investigación, desarrollo e innovación, así como un instrumento necesario para documentar la elaboración de políticas en ciencia y tecnología, así como la definición de prioridades para el desarrollo articulado de la ciencia, la tecnología y la innovación en una región, país, institución o cualquier otra instancia (Díaz, Guzman y Orea, 2007; Giráldez, Díaz y Armas, 2008).

La mayoría de las organizaciones regionales e internacionales (relacionadas con estudios de patentes) utilizan mayoritariamente los análisis sobre el desarrollo de los países indicadores, que reflejan las macroeconomías (cantidad de patentes solicitadas y concedidas por nacionales y extranjeros, etc.), mas no publican indicadores que representen los desarrollos internos (microeconomía) de los países, considerados estos últimos esenciales en la presente investigación para conocer los contextos donde se desarrollan las innovaciones endógenas. Ya que es tan importante conocer la productividad científico-tecnológica de un país, por ejemplo, estudiar el comportamiento de sus actores dentro del contexto nacional e internacional. Se precisa dejar 
atrás la concepción teoricista en la que la ciencia son las teorías y formulaciones teóricas, y la tecnología son patentes de artefactos materiales, conjeturando incluso que la tecnología es sólo ciencia aplicada. Tendencia incierta, hoy los márgenes entre la ciencia y la tecnología se tornan cada vez más difusos y difícilmente observables, por resulta tan complejo definir indicadores que reflejen fielmente la realidad científico tecnológica y de innovación de un dominio.

Tal y como existen metodologías para el análisis del sistema de ciencia, basadas en indicadores de producción científica (artículos), existen metodologías para medir o evaluar el desarrollo tecnológico (Meyer et al., 2004). Para este fin, las patentes son la fuente de información más confiable y relevante que permite medir la producción tecnológica de un dominio (Medina, 1996). Los estudios más significativos sobre la importancia del uso métrico de la información de patentes empezaron a publicarse en los sesenta. En los ochenta, Narin, Hamilton y Olivastro (1995) hablan ya de la patentometría como un equivalente del análisis bibliométrico de patentes, convirtiéndose, desde entonces, en uno de los análisis de mayor utilidad para las industrias, porque permiten un mayor acercamiento a la realidad tecnológica; así como identificar nichos tecnológicos de industrias y firmas (Stuart y Podolny, 1996); identificar redes de innovación en el desarrollo de tecnologías (Griliches, 1990) y posibles relaciones entre sí (ciencia-tecnología) (Brooks, 1994). Además de utilizar las patentes para evaluar las investigaciones, la evolución de las tecnologías y el desarrollo de diferentes dominios (Trajtenberg, 1990; Jaffe, Trajtenberg y Henderson, 1993; Narin, 1994; 1998).

A partir de todas las fortalezas de las patentes como fuente de información y las posibilidades que ofrece para describir el desarrollo tecnológico de los países, se identifican como una de las vías más expeditas y efectivas para describir la gran variedad de aspectos presentes en los procesos de innovación tecnológica regionales. Ante esta realidad, se manifestó, desde finales del siglo XX, la necesidad de perfeccionar los estudios métricos y las propuestas de nuevos métodos de análisis y medición de la información contenida en los documentos de patentes, las cuales permiten describir los principales comportamientos de un país, proyecto, línea de investigación u otro dominio tecnológico, saber, además, las relaciones que se establecen entre los titulares e inventores más productivos por sectores de la tecnología.

También son necesarios los estudios que analicen la descomposición de la clasificación por los sectores técnicos más desarrollados, analizando sus relaciones por años, titulares, inventores, e identificando el núcleo, especialización y diversidad tecnológica por ramas del conocimiento tecnológico. Es- 
del país, así como las relaciones multi e interdisciplinares en los resultados científicos y tecnológicos alcanzados, indagando además en las relaciones de triple hélice que se establezcan en los contextos tecnológicos.

Sin embargo, la revisión de la bibliografía consultada mostró que, hasta la fecha, no existen muchos estudios patentométricos que obtengan todos los análisis ya mencionados. El estado de la cuestión reveló pocas investigaciones que manejen metodologías que utilicen técnicas de redes sociales e indicadores relacionales y complejos para describir las diferentes dimensiones del comportamiento tecnológico de un dominio; país, región, línea de investigación, proyectos de investigación, entre otros.

Los estudios patentométricos no se aplican óptimamente en todos los países ni por parte de los organismos internacionales, tampoco se aprovechan todas las potencialidades que ofrecen estos estudios para conocer los diferentes contextos de las innovaciones tecnológicas de un país. Este trabajo tiene como objetivo analizar el comportamiento métrico de las patentes concedidas en Cuba, aplicando una metodología propia que describe las principales innovaciones científico tecnológicas patentadas por la Oficina Cubana de Propiedad Industrial.

\section{Material y MÉTODOS}

Las patentes se consideran unidades de capacidad tecnológica porque representan conocimiento tecnológico, identificándolas como unidades de estudio, cómputo y medición sobre las que se trabajará aquí. Y como fuente de datos se establecen las patentes concedidas por la Oficina Cubana de la Propiedad Industrial de Cuba (OCPI), publicadas en la base de datos de esta oficina y en su boletín oficial.

Se descargaron, sin fines de lucro, todas las patentes registradas en la OCPI y publicadas en su boletín oficial entre el 1 de enero de 1997 y el 31 de diciembre de 2008, es decir, en un periodo de doce años. Del total de patentes descargadas, sólo fueron introducidas a la base de datos los documentos con toda la información solicitada en los campos obligatorios de la patente.

El periodo que se analiza es una serie cronológica de doce años, enmarcada entre 1997 y 2008. La fecha que se recupera es el año de concesión de la patente. Se excluye la fecha de solicitud de la patente, considerando más apropiado en esta investigación las fechas de las patentes concedidas, porque representan conocimiento nuevo constituido e introducido en el mercado internacional.

Se utiliza el software proIntec para la descarga, normalización, procesamiento, análisis y visualización de los datos procedentes del documento de 
patente; software con confiabilidad probada y contrastada en diferentes estudios internacionales (Moya et al., 2011; Pérez, Díaz y Giráldez, 2014; Díaz et al., 2014d; Díaz et al., 2014a; Díaz et al., 2014b; Díaz et al., 2014c; Díaz et al., 2016).

Y se manipulan como técnica de análisis y visualización de datos las redes presentadas en forma de nodos o vértices, en las que la estructura semántica se encuentra definida por los enlaces o relaciones que conectan dichos nodos. La visualización mediante redes hace posible representar las conexiones en red, se utilizan variables cruzadas para identificar patrones que subyacen en las redes de innovación y describir los comportamientos más significativos de cada contexto tecnológico. En tanto que para la visualización de la información en forma de mapas tecnológicos se utiliza el algoritmo Pathfinder, para mostrar sólo los enlaces más relevantes de las redes mapeadas. Se usa como técnica de poda la delimitación numérica de la intensidad de las relaciones en los nodos cuya relación es débil. En cuanto al posicionamiento de los nodos, se emplea el algoritmo de representación espacial Spring Embedded, y el Netdraw para manipular los gráficos en las representaciones visuales.

\section{ResUlTADOS Y DISCUSIÓN}

Después del procesamiento realizado a los registros de patentes, se obtuvo un total 901 patentes concedidas por la OCPI entre 1997 y 2008. De esta cifra, existe un total de 542 patentes en los doce años estudiados cuyos titulares son cubanos (Figura 1).

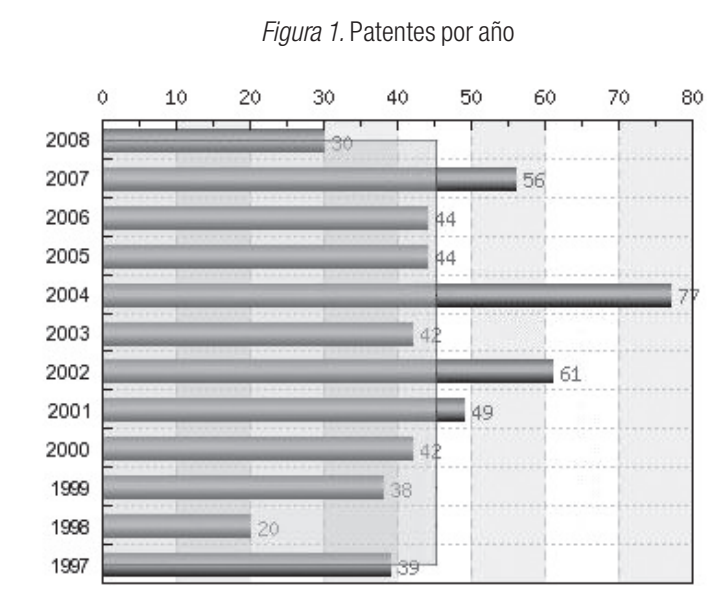


El análisis por secciones de la Clasificación Internacional de Patentes (CIP), o secciones del conocimiento tecnológico, reveló que Cuba tiene invenciones en las ocho secciones de la técnica; y que existen diferentes autores (investigadores e inventores) e instituciones (titulares) trabajando las mismas secciones del conocimiento tecnológico, e incluso trabajando de conjunto. Mas no todos los sectores de innovación poseen los mismos grados de maduración tecnológica, hay sectores del conocimiento tecnológico con mayor capacidad innovadora que otros. En los sectores del conocimiento que obtienen mayores resultados tecnológicos innovadores se encuentran: Química-Metalúrgica (C-222); Necesidades Corrientes de la Vida (A-212 patentes); la Física (G-58 patentes) y las Técnicas Industriales Diversas-Transporte (B54 patentes).

El Cuadro 1 se obtuvo cruzando la variable patente por años con la variable clasificación temática, el análisis de este indicador revela la trayectoria tecnológica de las innovaciones cubanas; refleja las nuevas tendencias que emergen en el desarrollo tecnológico del país en los últimos años, así como los sectores temáticos donde existen innovaciones permanentes.

Este análisis reveló que Cuba investiga en todas las áreas del conocimiento tecnológico de forma sistemática, o sea el país tiene una estrategia sostenida en el desarrollo de sus tecnologías. Se infiere que existe una permanencia en la introducción y escalado de innovaciones en el mercado internacional, convirtiendo el país en un fuerte competidor en el desarrollo de determinadas tecnologías, principalmente médicas, como develó el análisis por sectores temáticos (Lage, 2008).

Cuadro 1. Patentes cubanas por sección de la técnica por años

\begin{tabular}{|l|l|l|l|l|l|l|l|l|l|}
\hline $\begin{array}{c}\text { CIP/ } \\
\text { Años }\end{array}$ & A & B & \multicolumn{1}{|c|}{ C } & D & E & F & G & H & Total \\
\hline 1997 & 16 & 4 & 17 & & 1 & 3 & & & 41 \\
\hline 1998 & 7 & 5 & 4 & & 1 & 2 & 1 & 1 & 21 \\
\hline 1999 & 9 & 2 & 11 & & 3 & 4 & 4 & 7 & 40 \\
\hline 2000 & 16 & 5 & 12 & & & & 13 & 3 & 49 \\
\hline 2001 & 21 & 3 & 16 & & 1 & 3 & 6 & 2 & 52 \\
\hline 2002 & 22 & 2 & 32 & 1 & & 5 & 5 & & 67 \\
\hline 2003 & 17 & 5 & 23 & 1 & 2 & 1 & 6 & & 55 \\
\hline 2004 & 42 & 8 & 27 & 1 & & 4 & 9 & 3 & 94 \\
\hline 2005 & 17 & 3 & 19 & & 2 & 4 & 4 & & 49 \\
\hline
\end{tabular}




\begin{tabular}{|l|l|l|l|l|l|l|l|l|l|}
\hline $\begin{array}{c}\text { CIP/ } \\
\text { Años }\end{array}$ & \multicolumn{1}{|c|}{ A } & B & \multicolumn{1}{|c|}{ C } & \multicolumn{1}{|c|}{ D } & E & F & G & H & Total \\
\hline 2006 & 18 & 7 & 15 & 3 & 1 & 2 & 4 & 1 & 51 \\
\hline 2007 & 21 & 5 & 31 & 1 & & 1 & 4 & 3 & 66 \\
\hline 2008 & 6 & 5 & 15 & 1 & & 4 & 2 & & 33 \\
\hline Total & 212 & 54 & 222 & 8 & 11 & 33 & 58 & 20 & 618 \\
\hline
\end{tabular}

Leyenda: Los títulos de las secciones son los siguientes:

A) Necesidades corrientes de la vida; B) Técnicas industriales diversas; transportes; C) Química; metalurgia; D) Textiles; papel; E) Construcciones fijas; F) Mecánica; iluminación; calefacción; armamento; voladura; G) Física; H) Electricidad.

Fuente: software prolntec.

La Figura 2 muestra las empresas titulares extranjeras con más de dos patentes registradas en Cuba. La mayor presencia la ocupa la firma Bayer Healthcare de Alemania (subgrupo de Bayer, proveedor de productos médicos y farmacéuticos) que investiga, desarrolla, fabrica y comercializa productos innovadores para la prevención, diagnóstico y tratamiento de enfermedades. Le siguen las firmas estadounidenses Pfizer, Warner-Lamber Company y la holandesa Heineken. Pfizer es una de las compañías biofarmacéuticas más importantes del mundo (más de siglo y medio) en descubrimiento, desarrollo y fabricación de medicamentos para personas y animales.

Figura 2. Titulares de otros países con más de dos patentes en Cuba

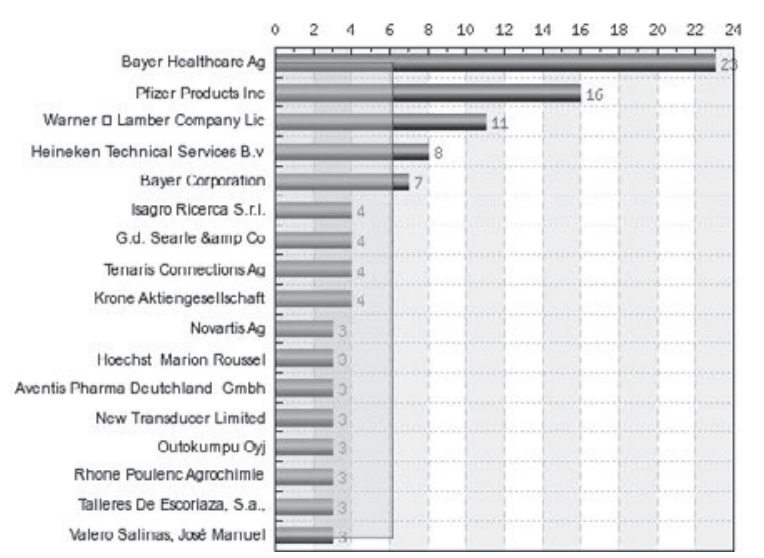

Fuente: software prolntec.

La biotecnología es uno de los ejemplos más ilustrativos: Europa publica la mayor producción científica sobre esta temática, siendo superior a la de Es- 
que los europeos mismos. Informes de la Comisión Europea dicen que este continente supera a Estados Unidos y Japón en la producción científica (González, 2002; Hidalgo, 2003). Y que las universidades europeas son excelentes generadoras de conocimiento, pero el nivel de rendimiento europeo en el sector de la alta tecnología ha decrecido, lo que se manifiesta en un incremento en el déficit comercial de este tipo de productos (Comisión Europea, 2003; Azagra, Caracol y Llerena, 2006). Mientras en Cuba la política científica tecnológica es diferente, por ejemplo, en las universidades se les exige mediante el Sistema Interno de Propiedad Industrial (SIPI) una integración adecuada entre la gestión de la ciencia, la tecnología y la innovación tecnológica con la gestión de la propiedad industrial, manejando articulada y conjuntamente ambos fenómenos en el desarrollo de los proyectos de innovación tecnológica $(\mathrm{I}+\mathrm{D}+\mathrm{i})$.

Este escenario cubano sería cautivador para países con intereses tecnológicos similares, incluso constituir una fuerte competencia principalmente en tecnologías biotecnológicas, razón por lo que constituye un destino obligado para proteger patentes de este sector y otras áreas a fines (Lage, 2004: 1039). El análisis de las patentes foráneas dejó ver algunos datos muy interesantes, por ejemplo, Estados Unidos es el país que tiene registradas el mayor número de patentes foráneas en Cuba y además es el único titular extranjero con registros en todas las secciones del conocimiento de la técnica; y España es el país que más aporta conocimientos al país mediante investigaciones bajo titularidad cubana, con una presencia sostenida en casi todos los años estudiados.

Un análisis infaltable en esta investigación es el cálculo de la productividad por titulares. En primera instancia, se conoció que existen 191 titulares cubanos que han registrado sus patentes entre 1997 y 2008. La Figura 3 filtra los titulares más productivos (más de tres patentes en el periodo estudiado), representando la cantidad de patentes por años de cada titular. Según este análisis, el núcleo de titulares más productivos de Cuba procede de universidades y centros de investigación, se estima que estas instituciones tienen una estrategia de patentamiento emprendedora en los procesos de ciencia e innovación tecnológica. 
Figura 3. Cantidad de patentes por titulares y años

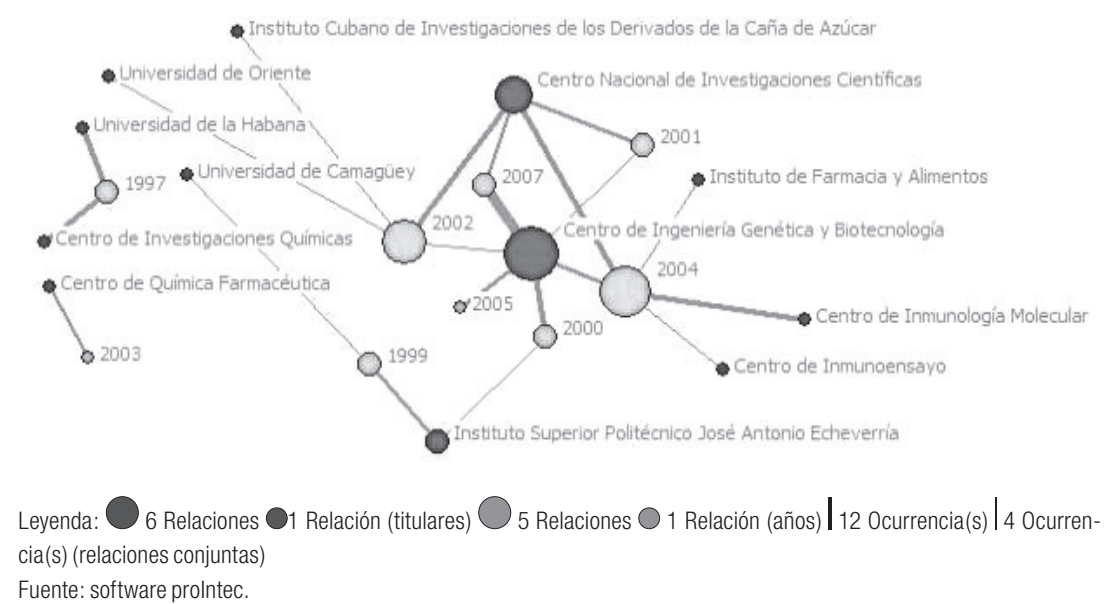

La Figura 3 posiciona al Centro de Ingeniería Genética y Biotecnología (CIGB) como el titular con mayor cantidad de patentes y presencia tecnológica en casi todos los años estudiados, exhibiendo su mayor productividad en el año 2007 con 12 patentes concedidas. El CIGB es una institución del polo científico con más de 130 patentes solicitadas en Cuba, 75 invenciones registradas en otros países, y más de mil solicitudes en todo el mundo (OMPI2009). El Centro Nacional de Investigaciones Científicas (cnic) ocupa la segunda posición dentro del país. Este centro ha sido multiplicador de un gran número de doctores en ciencia en el país, además de haber sido gestor y formador de varios centros a partir de los grupos de investigación que creó e impulsó, por ejemplo, el Centro de Inmunoensayo (CIE); Centro de Química Farmacéutica (CQF), Centro de Neurociencias, etc. Le sigue en número de patentes la Universidad de La Habana, con su mayor productividad en 1997, cuando le otorgaron siete patentes. Esta universidad con más de 285 años de fundada, ha sido el centro que tradicionalmente publica el mayor porcentaje de artículos científicos en corriente principal (Arencibia y Moya, 2008). En este caso, se corresponde su productividad científica con la tecnológica, al ocupar la tercera posición dentro de los titulares más productivos de Cuba. Esto indica que, a pesar de ser una universidad, continúan las investigaciones en determinados sectores del conocimiento tecnológico hasta cerrar el ciclo de $\mathrm{I}+\mathrm{D}+\mathrm{i}$, e introducir los resultados de sus investigaciones científico-tecnológicas en la economía y en la sociedad.

En cambio, el Centro de Inmunología Molecular, con su mayor presencia en 2004, tiene como principal misión obtener y producir nuevos biofármacos 
destinados al tratamiento del cáncer y otras enfermedades crónicas no transmisibles. Tiene más de 173 solicitudes de patentes presentadas en países extranjeros, 82 de las cuales concedidas en 27 países.

El análisis de la productividad por inventores develó que existen 1,684 investigadores vinculados a la generación de tecnologías en el periodo que se analiza. Como es un grupo muy grande de personas, se determinó trabajar sólo con los actores que tienen más de tres patentes en un año. Esta estrategia de selección se considera rigurosa, por las grandes diferencias que presenta la patente como documento científico técnico y legal del resto de la literatura científica. A partir de esta premisa, se infiere que los inventores-actores que se obtengan tras esta poda representarán los científicos más productivos y tecnólogos más eminentes del país, conformando el principal núcleo de investigación cubano (Figura 4).

Figura 4. Inventores por principales clases temáticas (CIP)

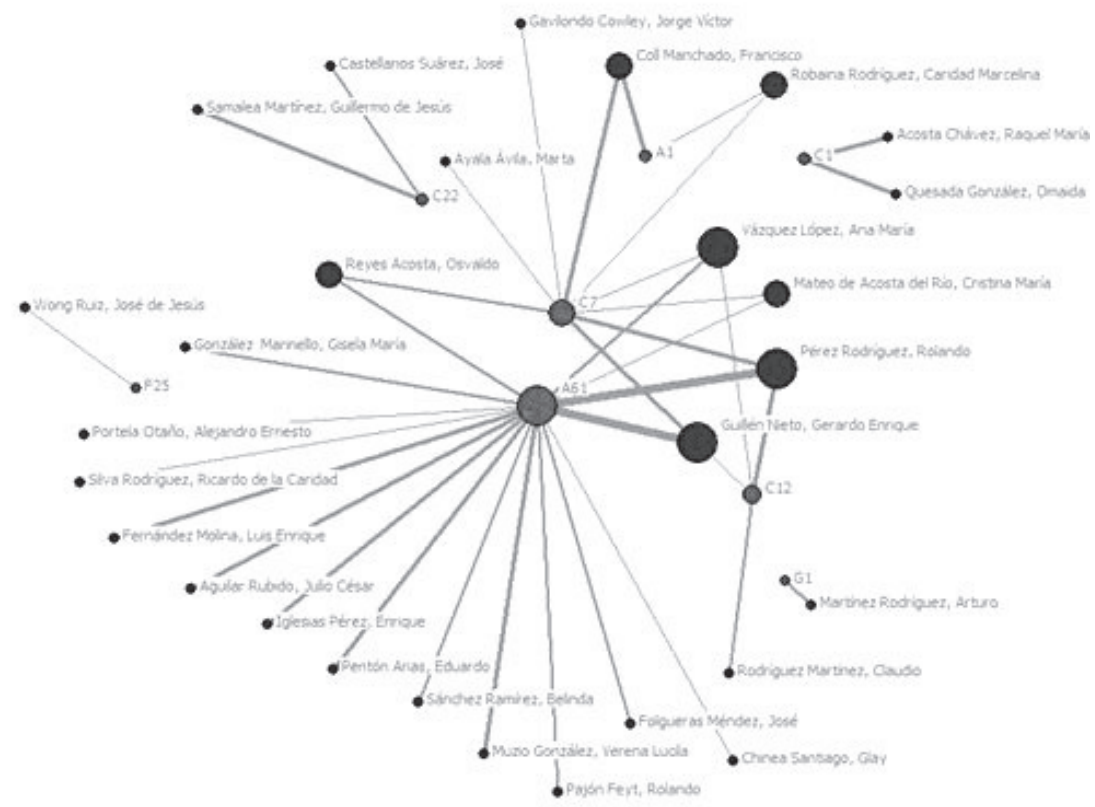

Leyenda: 3 Relaciones 1 Relación (titulares) $\bigcirc 17$ Relaciones $\bigcirc 1$ Relación (CIP) $\mid 14$ 0currencias $\mid 4$ 0currencias (relaciones conjuntas).

Fuente: software prolntec.

Los análisis de colaboración en el desarrollo de las investigaciones tecnológicas es una dimensión muy valiosa dentro de los estudios patentométricos, 


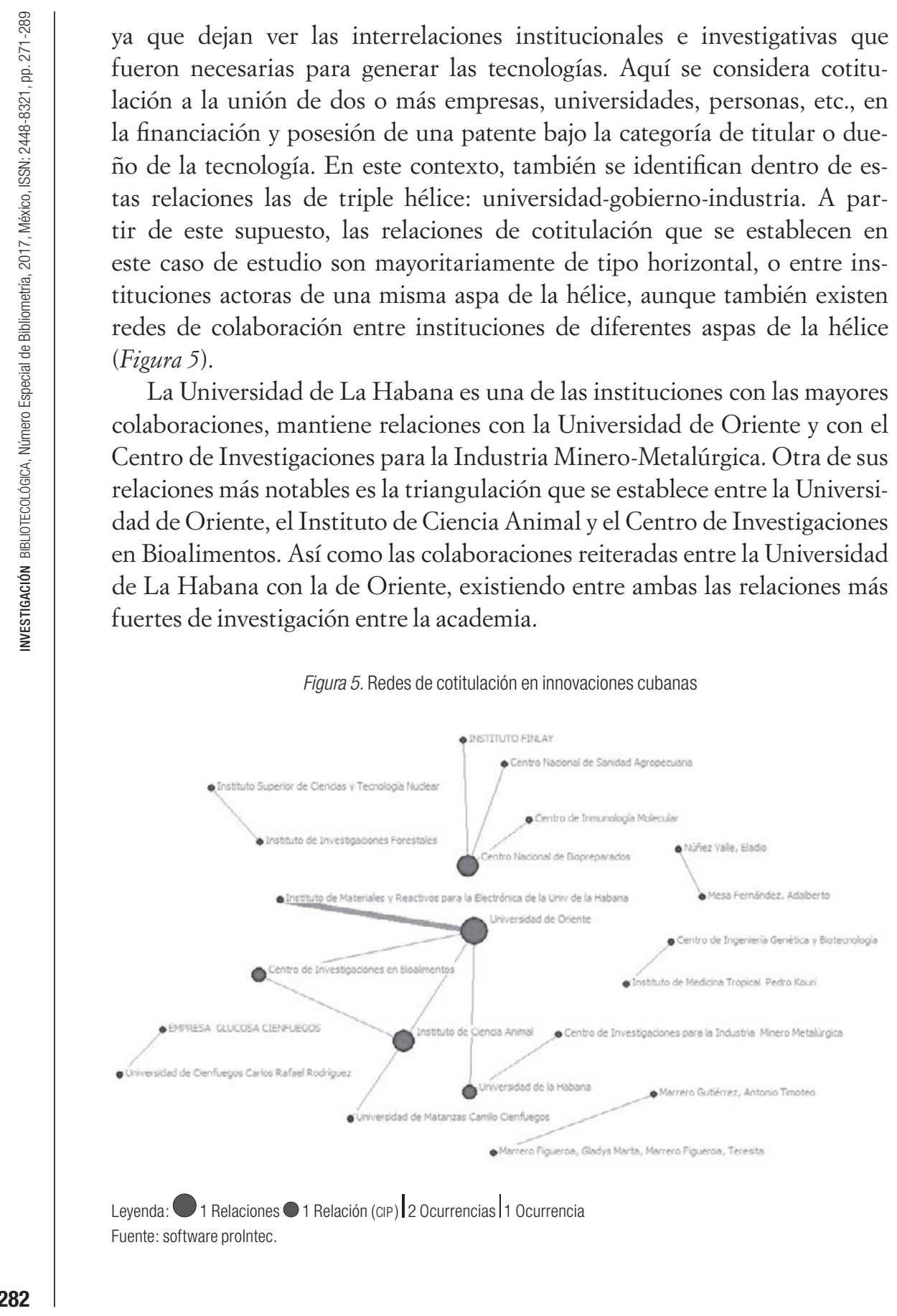


El Centro Nacional de Biopreparados es la institución que más ha trabajado en colaboración (Figura 5), es considerado el titular que mayores potencialidades tiene para establecer relaciones de investigación colaborativas aprovechando mejor sus capacidades tecnológicas.

Otra relación significativa se establece entre el CIGB y el Instituto Pedro Kourí en el área de la biotecnología, así como entre el Instituto de Investigaciones Forestales y el Instituto Superior de Ciencias y Tecnología Nuclear. Se consideran también muy interesantes las colaboraciones que se gestan en Cuba entre los ámbitos universidad-empresa. Estos resultados son fruto de la estrategia que ha establecido la política nacional de ciencia y tecnología del país para articular mejor los recursos financieros destinados a la investigación desarrollo e innovación tecnológica en función de los principales problemas de la sociedad cubana (CITMA, 2012).

Es un hecho que los tecnólogos cubanos no investigan lo que quieren, sino lo que más necesita el país; son los programas nacionales y territoriales de ciencia y tecnología los que establecen las pautas de la investigación en Cuba (CITMA, 2014; CIEM, 2012). Muy al contrario de otros países, donde no se tiene claro el destino de las invenciones y los beneficiarios de los principales desarrollos tecnológicos de los países (Aboites, Beltrán y Guzman, 2008).

Esta investigación igualmente analiza las relaciones colaborativas entre los inventores, desde la perspectiva de que existen diferentes tipos de redes en el contexto de las innovaciones. En este caso de estudio, se analizaron las redes que se construyeron entre los actores investigadores para el desarrollo de determinadas tecnologías.

Por la gran cantidad de inventores cubanos que existen en el periodo estudiado, se determinó podar el análisis, estableciendo como mínimo dos relaciones. La Figura 6 muestra los actores investigados conjuntamente en más de dos ocasiones, identificando incluso en este mapa algunos grupos de investigación con hasta seis relaciones de colaboración en el desarrollo de diferentes tecnologías. Lo que constata que en el país existen varios grupos de investigación que trabajan conjuntamente en la generación de innovaciones tecnológicas. Se considera que este tipo de análisis es muy valorado, cuando se necesita conocer los principales frentes de investigación de un dominio así como las relaciones entre sí; de hecho, la Figura 6 refleja los principales núcleos de investigación que tiene el país en el periodo estudiado. 


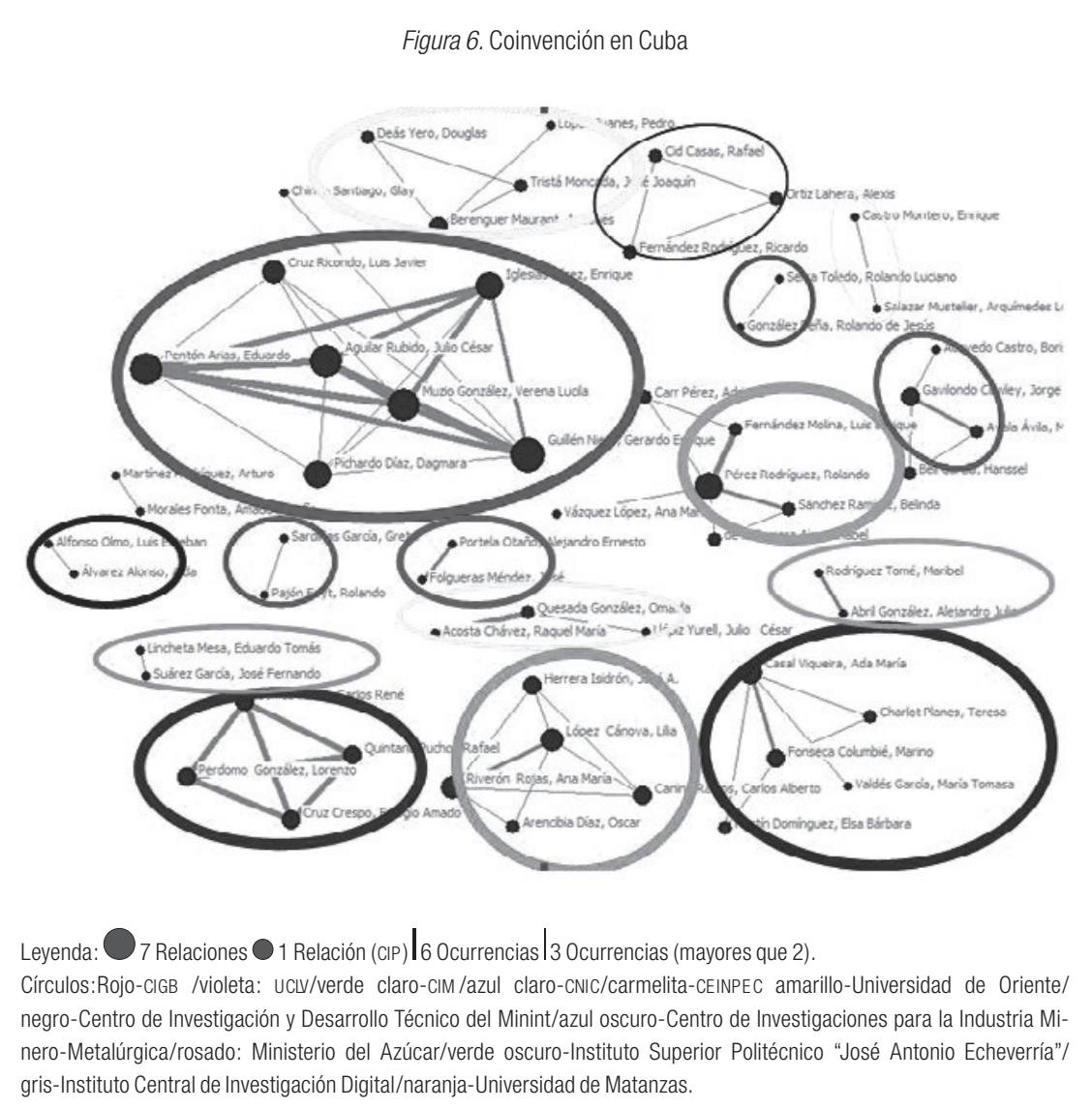

La Figura 7 muestra la procedencia de los inventores-actores que han trabajado coordinadamente con investigadores cubanos. Este análisis representa una amplia colaboración entre los actores que generan las patentes, corroborando las relaciones de Cuba con actores de otros países como España, México, Brasil, Alemania, Canadá, Argentina, entre otros.

Otro indicador importante en este tipo de estudios es el relacionado con la capacidad de captura de conocimiento tecnológico dentro del grupo de indicadores que reflejan el adelanto científico tecnológico de un país. Aquí se calcula la cantidad de patentes de inventores no nacionales y titulares nacionales, con la cantidad de patentes de titulares nacionales por años. En este caso de estudio, se modificó este indicador y lo que se calculó fue la captura detallada por país de procedencia del titular, cuyo análisis extrajo los siguientes datos: 
España tiene el mayor aporte de conocimiento a investigaciones bajo titularidad cubana, con una presencia sostenida en casi todos los años estudiados.

- México tiene gran participación en 2008.

- Canadá con los valores más altos en 2004.

- y otros países como Argentina, Israel, Rusia, etc., han trabajado en colaboración con Cuba.

- Mientras los niveles más bajos de colaboración internacional son con Inglaterra, Estados Unidos, Francia y Alemania.

Figura 7. Coinvención por procedencia del país

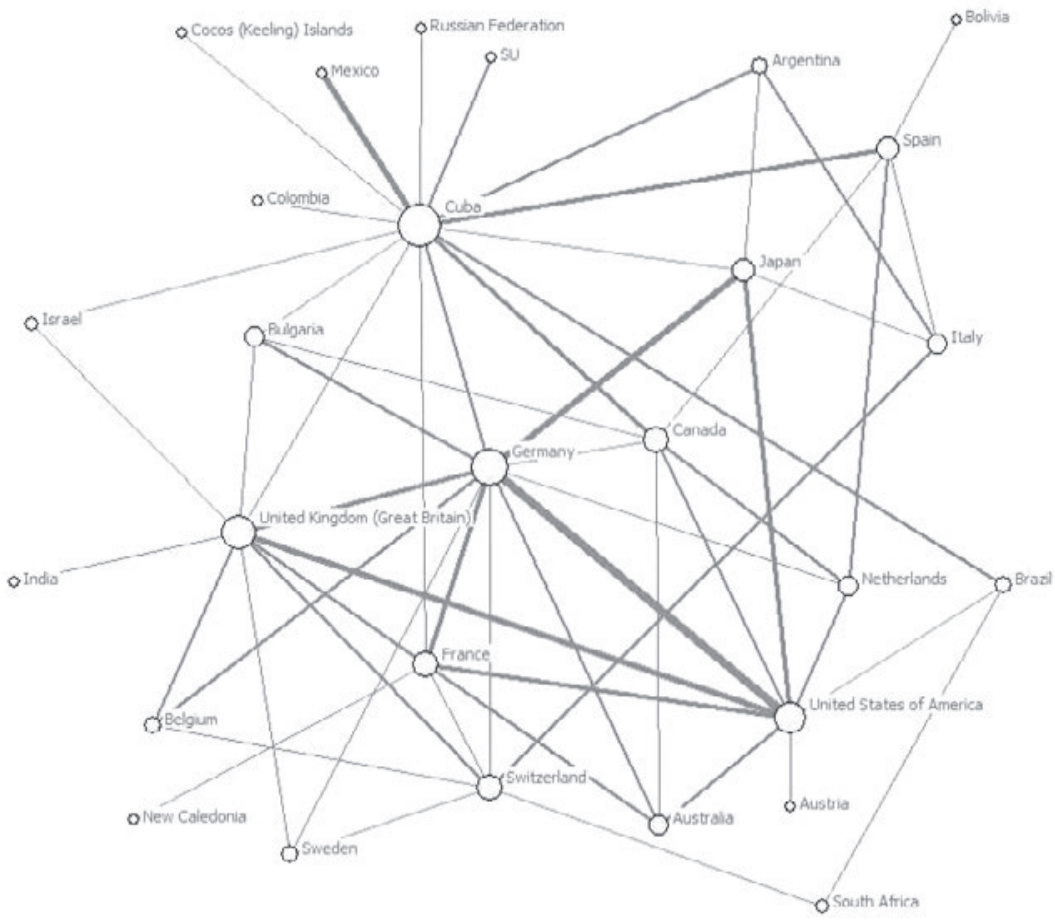

Leyenda:

15 relaciones $\bigcirc 1$ (países) relación $\mid 11$ ocurrencias $\mid 1$ ocurrencia (relaciones conjuntas)

Fuente: prolntec.

En el país existe un potencial científico muy alto, lo que significaría un peligro o alerta a otros países productores de similares tecnologías. Esta situación convierte al país en un frente tecnológico muy atractivo, tanto para desarrollar investigaciones en colaboración conjunta, como para proteger 
determinadas invenciones que pudieran ser copiadas o transferidas al sector nacional (Lage, 1995; 2001).

\section{Consideraciones finAles}

Del análisis del estado de la cuestión de esta investigación se infiere y constata que hoy se precisan instrumentos metodológicos más pragmáticos, un sistema de indicadores más confiable y técnicas métricas más precisas en los estudios patentométricos. Con la finalidad de obtener una mayor cantidad de datos sobre el contexto donde se realizan las innovaciones, principalmente el contexto social (investigadores e instituciones), contexto geográfico (países, ciudades, regiones, etc.), contexto temporal (series cronológicas), contexto temático (sectores del conocimiento tecnológico donde se han desarrollado las investigaciones), contexto relacional y participativo (relaciones de colaboración), entre los contextos que mayor información tributan a este tipo de investigaciones.

El estudio sobre el desarrollo tecnológico de Cuba, basado en el análisis de la información contenida en los documentos de patentes, mostró, de cierta forma, la correspondencia del comportamiento de este país con las nuevas tendencias teóricas sobre innovación. Ésta, como sistema, es un proceso social en el que convergen e interactúan distintos actores y factores, de diferente naturaleza, en un entorno que propicia esta integración, siendo de un valor mayor la inclusión de cada una de sus partes y no su simple suma, obteniendo de esta integración sistémica un estadio superior de desarrollo en la solución de los problemas de la sociedad. Supuesto que ha sido validado en este caso de estudio.

Otra consideración importante es que el estudio reveló que las universidades con mayor producción científica en Cuba se corresponden con las de mayor productividad tecnológica o de patentes, lo que indica que existen universidades cubanas que cierran el ciclo de investigación, desarrollo e innovación. Este escenario innovador favorece los procesos de aprendizaje colectivo, en los que fluye el conocimiento desde diferentes ambientes, propiciando la transferencia de conocimiento y la creación de habilidades y capacidades tecnológicas. Se muestra, por parte de Cuba, una alta capacidad de retención y captura de conocimiento tecnológico, con una estrategia de patentamiento efectiva en el ámbito de la colaboración internacional.

Este análisis de patentes permitió comprender que Cuba tiene un desarrollo tecnológico con alta escalabilidad de sus innovaciones en el mercado nacional e internacional, radicando su vanguardia tecnológica principalmente en el área de la biotecnología. 


\section{Reconocimientos}

Se reconoce el apoyo del Programa VLIR, Red Universitaria de Cooperación del Consejo Inter Universitario Flamenco (VLIR), específicamente al proyecto International Projetc "Fortalecimiento del papel de las tic en las universidades cubanas para el desarrollo de la sociedad", Cuba-Bélgica, en el desarrollo de esta investigación.

\section{REFERENCIAS}

Aboites, J, T Beltrán y A. Guzman. 2008. “¿Quién apropia el conocimiento producido por los inventores?” La Habana: IV Seminario Internacional de Estudios Cuantitativos y Cualitativos de la Información INFO.

Arencibia, Ricardo y Felix Moya-Anegón. 2008. "Visibilidad e impacto de las instituciones adscritas al Ministerio de Educación Superior de Cuba en la Web de la Ciencia (2004-2006)". La Habana: IV Seminario Internacional de Estudios Cuantitativos y Cualitativos de la Información INFO.

Azagra-Caro, J.M., M Caracol y P. Llerena. 2006. "Patent production at a European Research University: exploratory evidence at the laboratory level", Journal of Technology Transfer, vol 31:257-268.

Brooks, Dana. 1994. "The relationship between science and technology", Research Policy, vol. 23: 477-86.

CIEM. 2012. "Investigación sobre desarrollo humano y equidad en Cuba". La Habana: Caguayo.

CITMA. 2014. "Programas para el perfeccionamiento y desarrollo de la innovación en Cuba". La Habana: CITMA.

CITMA. 2012. "Bases para el perfeccionamiento y desarrollo de la innovación en Cuba”. La Habana: CITMA.

Comisión Europea. 2003. "El rendimiento de la investigación de la ue sigue aumentando, pero se enfrenta a importantes retos", Tercer Informe Europeo sobre Indicadores en Ciencia y Tecnología. Bruselas: CE.

Díaz Pérez, Maidelyn. 2007. "Producción tecnológica de América Latina con mayor visibilidad internacional: 1996- 2007”. Granada: Universidad de Granada, tesina en opción al Diploma de Estudios Avanzados.

Díaz Pérez, Maidelyn. 2005. "La información de patentes en el ciclo de vida de un proyecto de investigación: caso de estudio". La Habana: Universidad de La Habana, Facultad Comunicación e Información Científica, Maestría en Ciencias de la Información.

Díaz Pérez, Maidelyn, Raudel Giráldez Reyes, Félix Moya Anegón y Humberto Carrillo-Calvet. 2016. "Análisis patentométrico de un dominio geográfico: Iberoamérica”. Transinformação, vol. 28, no. 3.

Díaz Pérez, Maidelyn y Félix Moya Anegón. 2008. "El análisis de patentes como estrategia oportuna para la toma de decisiones innovadoras", El Profesional de La Información, vol. 17, no. 1. 
Díaz Pérez, Maidelyn, María Victoria Guzman y Ubaldo Orea. 2007. "Estudio patentométrico de un proyecto de investigación”, Revista Ciencias de la Información, vol. 38, no. 3: 57-66.

Díaz Pérez, Maidelyn, María Victoria Guzman, Raudel Giráldez Reyes, Dayron Armas Peña, Reinaldo Javier Rodríguez Font y Humberto Carrillo-Calvet. 2014a. "Tuberculosis, Bacillus Calmette-Guérin (BCG) y vacunas de tuberculosis: análisis de patentes". Revista Cubana de Información en Ciencias de la Salud, vol. 25, no. 3.

Díaz Pérez, Maidelyn, Raudel Giráldez Reyes, Dayron Armas Peña y Reinaldo Javier Rodríguez Font. 2014b. "El profesional de la información en los procesos de innovación de la educación superior”, Revista Caribeña de las Ciencias Sociales (noviembre).

Díaz Pérez, Maidelyn, Raudel Giráldez Reyes, Dayron Armas Peña, Reinaldo Javier Rodríguez Font y María Rosa Govea González. 2014c. "La multi e interdisciplinaridad en la generación de tecnologías en Cuba", Avances, vol. 17, no. 1.

Díaz Pérez, Maidelyn, Raudel Giráldez Reyes, Dayron Armas Peña, Reinaldo Javier Rodríguez Font, Elio Atenógenes Villaseñor García y Humberto Carrillo-Calvet. 2014d. "Tecnologías constituidas, innovaciones en proceso y tecnologías introducidas en el mercado internacional de un dominio tecnológico: caso de estudio", TransInformação, vol. 26, 3 .

Díaz Pérez, Maidelyn, Soleidy Rivero y Felix Moya Anegón. 2010. "Producción tecnológica latinoamericana con mayor visibilidad internacional: 1996-2007. Un estudio de caso: Brasil", Revista Española de Documentación Científica, vol. 33, no, 1:34-62.

Giráldez Reyes, Raudel, Maidelyn Díaz Pérez y Dayron Armas Peña. 2008. "ProInTec: un software para el tratamiento inteligente de datos sobre patentes", Acimed, vol. 17, no. 5 .

González, G. 2002. "Los servicios de información sobre propiedad industrial. Constitución, actualización y promoción. experiencia española”, Conferencia Nacional de la Organización Mundial de la Propiedad Intelectual.

Griliches, Zvi. 1990. "R\&D, patent statistics as economic indicators", Journal of Economic Literature, 28, no. 4: 1661-1797.

Hidalgo, A. 2003. "Las capacidades de innovación tecnológica en españa a través del análisis cualitativo de las patentes", X Seminario Latino-iberoamericano de Gestión Tecnológica ALTEC 2003, "Conocimiento, innovación y competitividad: Los desafíos de la globalización”.

Jaffe, Adam B, Manuel Trajtenberg y Richard Henderson. 1993. "Geographic localization of knowledge spillovers as evidenced by patent citations", Quarterly Journal of Economics, 108, no. 3: 577-598.

Lage Dávila, Agustín. 2008. "Ciencia y soberanía: los retos y las oportunidades”, Reflexiones sobre el desarrollo de la biotecnología en Europa y América Latina, SELA.

Lage Dávila, Agustín. 2004. Propiedad y expropiación en la economía del conocimiento. gestión del conocimiento. Concepto, aplicaciones y experiencias. La Habana: Academia.

Lage Dávila, Agustín. 2001. "La ciencia y la cultura: las raíces culturales de la productividad”, Educación Médica Superior, vol. 15, no. 2: 24-25.

Lage Dávila, Agustín. 1995. "Desafíos del desarrollo. El problema de las nuevas funciones de la investigación en la sociedad, visto desde la perspectiva de un hombre de laboratorio y en un país en desarrollo", Ciencia, Innovación y Desarrollo, vol. 1, no. 1. 
Medina, Mario. 1996. "De la práctica de los indicadores de la ciencia y la tecnología a la configuración del desarrollo tecnocientífico", en Hacia la construcción de un Observatorio de Ciencia y Tecnología. Bogotá: Instituto Colombiano para el Desarrollo de la Ciencia y la Tecnología (Colciencias).

Meyer, Martin, Tiago Santos Pereirac, Olle Persson y Ove Granstrand. 2004. "The scientometric world of Keith Pavitt. A tribute to his contributions to research policy and patent analysis", Research Policy, vol. 33: 1405-1417.

Moya Anegón, Félix, Zaida Chinchilla Rodríguez, E. Corera Álvarez y Maidelyn Díaz Pérez. 2011. "Estudio de la producción científica y tecnológica en colaboración universidad-empresa en Iberoamérica", Red Emprendia.

Narin, Francis. 1998. "Patents and publicly funded research", en Assessing the Value of Research in the Chemical Sciences. Washington, D.C.: National Academy Press, 59-72.

Narin, Francis. 1994. "Patent bibliometrics", Scientometrics, vol. 30, no. 1: 147-155.

Narin, Francis, Kimberly S. Hamilton y Dominic Olivastro. 1995. "Linkage between agency supported research and patented industrial technology", Research Evaluation, vol. 5: 183-187.

Organización Mundial de la Propiedad Intelectual (OMPI). 2009. "Training course on intellectual property strategies in the health and pharmaceutical sector". Ginebra: OMPI.

Pérez Arreortúa, Noé, Maidelyn Díaz Pérez y Raudel Giráldez Reyes. 2014. "Análisis de contenido del dominio tecnológico 'Vegetable Oil Combustion"”, TransInformação, vol. 26, no. 3 .

Stuart, Toby E. y Joel M Podolny. 1996. "Local search and the evolution of technological capabilities”, Strategic Manage, vol. 17: 21-38.

Trajtenberg, Manuel. 1990. "A penny for your quotes: patent citations and the value of innovations". RAND Journal of Economics, vol. 21, no. 1: 172-187.

Para citar este texto:

Díaz Pérez, Maidelyn; Giráldez Reyes, Raudel; Carrillo Calvet, Humberto Andrés. 2017. "Comportamiento métrico de las patentes concedidas en Cuba: su contribución a la innovación tecnológica nacional". Investigación Bibliotecológica: Archivonomía, Bibliotecología e Información (Número Especial de Bibliometría): 271-289.

http://dx.doi.org/10.22201/iibi.24488321xe.2017.nesp1.57893 